\title{
Comparação de modelos estatísticos para estimativa da biomassa de árvores, e estimativa do estoque de carbono acima do solo em Cerrado
}

\author{
Comparison of statistical models for the estimation of tree biomass and for the estimation of \\ carbon stock above the soil in he Cerrado biome
}

\author{
Camila Paula de Oliveira ${ }^{\mathrm{I}}$, Márcio Rocha Francelino ${ }^{\mathrm{II}}$, Mayara Daher ${ }^{\mathrm{III}}$, Paulo Sérgio dos Santos Leles ${ }^{\mathrm{IV}}$, \\ Fernando Canto de Andrade ${ }^{\mathrm{V}}$
}

\begin{abstract}
Resumo
Neste trabalho foram comparados diferentes modelos estatísticos para a estimativa da biomassa individual de árvores, e estimados os estoques totais de biomassa e de carbono acima do solo, nos três compartimentos (arbóreo, herbáceo-arbustivo e serapilheira) de um cerrado sensu stricto típico no oeste da Bahia. Foi realizado o inventário florestal em 20 parcelas de $1000 \mathrm{~m}^{2}(20 \mathrm{~m}$ x $50 \mathrm{~m})$. Para ajuste dos modelos estatísticos foram cortadas e pesadas 90 árvores de diferentes espécies e classes diamétricas. Foram ajustados modelos para o diâmetro medido a $30 \mathrm{~cm}$ do solo $\left(\mathrm{D}_{30 \mathrm{~cm}} \geq 5 \mathrm{~cm}\right)$ e diâmetro medido a $1,30 \mathrm{~m}$ do solo (DAP $\left.\mathrm{P}_{1,30 \mathrm{~m}} \geq 3,5 \mathrm{~cm}\right)$. A equação escolhida foi aplicada aos dados das árvores levantados no inventário florestal, e assim foram estimados os estoques por hectare e total no compartimento arbóreo. As amostragens dos compartimentos herbáceo-arbustivo e serapilheira foram realizadas por subparcelas fixas. Foram alocadas sistematicamente 20 subparcelas de $4 \mathrm{~m}^{2}(2 \mathrm{~m} \mathrm{x} 2 \mathrm{~m})$ para amostragem do compartimento herbáceo-arbustivo e 20 subparcelas de $1 \mathrm{~m}^{2}(1 \mathrm{~m} \mathrm{x} 1 \mathrm{~m})$ para amostragem da serapilheira. O estoque de carbono foi estimado por fator de conversão da biomassa. A equação proveniente do ajuste do modelo de Schumacher \& Hall (logarítmica) $\left(\mathrm{R}^{2}=93,53 \%\right.$; $\left.\mathrm{S}_{\mathrm{xy}}=44,92 \%\right)$ foi a escolhida para estimar o estoque de biomassa das árvores do cerrado estudado. A biomassa total acima do solo, considerando os três compartimentos, foi estimada em $18,77 \mathrm{Mg} \mathrm{ha}^{-1}$, o que corresponde a um estoque total de $8,67 \mathrm{Mg}$ ha $^{-1}$ de carbono. O compartimento arbóreo foi responsável pela maior parte dos estoques (58\%), seguido da serapilheira (26\%) e herbáceo-arbustivo (16\%).
\end{abstract}

Palavras-chave: Formações savânicas; Cerrado típico; Equações de biomassa; Solos arenosos

\begin{abstract}
In this work, different statistical models were compared for estimating individual tree biomass and the total biomass and carbon stocks above the soil, in the three compartments (arboreal, herbaceous-shrub and litter) of a typical Cerrado in the west of Bahia state, Brazil. The forest inventory was carried out in 20 plots of $1000 \mathrm{~m}^{2}(20 \mathrm{~m}$ x $50 \mathrm{~m})$. In order to fit the statistical models, 90 trees of different species and diameter classes were cut and weighed. Models were fitted for the diameter measured at $30 \mathrm{~cm}$ from the soil $(\mathrm{D} 30 \mathrm{~cm} \geq 5 \mathrm{~cm}$ ) and the diameter measured at $1.30 \mathrm{~m}$ from the soil (DAP1,30m $\geq 3,5 \mathrm{~cm}$ ). The equation chosen was applied to the data of the trees collected in the forest inventory, and thus, the stocks per hectare and the total in the tree compartment were estimated. Samplings of the herbaceous-shrub and litter compartments were performed by fixed subplots. For sampling of the herbaceous-shrub and litter compartments, 20 subplots of $4 \mathrm{~m}^{2}(2 \mathrm{~m} \mathrm{x} 2 \mathrm{~m})$ and 20 subplots of $1 \mathrm{~m}^{2}(1 \mathrm{~m} \times 1 \mathrm{~m})$ were systematically allocated, respectively. The carbon stock was estimated by the biomass conversion factor. The equation resulting from the adjustment of the Schumacher \& Hall (logarithmic) model $(\mathrm{R} 2=93.53 \%$; Sxy $=44.92 \%)$ was chosen to estimate the biomass stock of the studied Cerrado trees. The total biomass above the soil, considering the three compartments, was estimated at $18.77 \mathrm{Mg}$ ha- 1 , which corresponds to a Carbon total stock of $8.67 \mathrm{Mg}$ ha- 1 . The arboreal compartment was responsible for most of the stocks (58\%), followed by litter (26\%) and herbaceous-shrub (16\%).
\end{abstract}

Keywords: Savanna formations; Typical Cerrado; Biomass equations; Sand soil

\footnotetext{
${ }^{I}$ Engenheira Florestal, MSc., Departamento de Ciências Ambientais, Instituto de Florestas, Universidade Federal Rural do Rio de Janeiro, Rod. Br 465, km 07, CEP 23890-000, Seropédica (RJ), Brasil. camiflorestal@gmail.com (ORCID: 0000-0002-0535-0080)

${ }^{I I}$ Engenheiro Agrônomo, Dr., Professor do Departamento de Solos, Centro de Ciências Agrárias, Universidade Federal de Viçosa, Av. Peter Henry Rolfs, s/n, CEP 36570-900, Viçosa (MG), Brasil. marcio.francelino@gmail.com (ORCID: 0000-0001-8837-1372)

III Geógrafa, MSc., Doutora em Agronomia pelo Departamento de Solos, Centro de Ciências Agrárias, Universidade Federal de Viçosa, Av. Peter Henry Rolfs, s/n, CEP 36570-900, Viçosa (MG), Brasil. mayara.daher@gmail.com (ORCID: 0000-0003-4937-2289)

${ }^{\text {IV }}$ Engenheiro Florestal, Dr., Professor do Departamento de Silvicultura, Instituto de Florestas, Universidade Federal Rural do Rio de Janeiro, Rod. Br 465, km 07, CEP23890-000, Seropédica (RJ), Brasil. pleles@ufrrj.br (ORCID: 0000-0002-8393-6095)

$\checkmark$ Engenheiro Florestal, Instituto de Florestas, Universidade Federal Rural do Rio de Janeiro, Rod. Br 465, km 07, CEP 23890-000, Seropédica (RJ), Brasil.fernando.canto@gmail.com (ORCID: 0000-0001-8520-6136)
} 


\section{Introdução}

A história do uso e ocupação da terra no Cerrado revela hoje intensa pressão antrópica no bioma, onde o uso insustentável dos recursos ambientais trouxe elevado grau de perturbação, tornando o Cerrado, um dos biomas mais ameaçados do planeta. Nos últimos 40 anos, mais de $40 \%$ da área total do Cerrado foi completamente desmatada e convertida para uso antrópico intenso (DIAS, 2008). Notadamente, a degradação do bioma ocorreu e ainda ocorre mais intensamente na região do oeste baiano, especialmente na bacia do Rio São Francisco, sendo esta, a região que mais evolui no tocante à conversão de áreas naturalmente vegetadas no bioma Cerrado (BRASIL, 2011).

Por sua extensão, diversidade biológica e potencial hídrico, o Cerrado apresenta papel fundamental para a qualidade ambiental global (BRASIL, 2011). Compreende o segundo maior bioma brasileiro e a savana tropical mais biodiversa do mundo (KLINK; MACHADO, 2005). É responsável por funções indispensáveis à geração de serviços ambientais essenciais, dentre os quais destaca-se a capacidade de armazenar em sua biomassa expressivas quantidades de carbono, atuando como grande assimilador e acumulador do carbono atmosférico. Deste modo, fica evidente a importância de estudar e conhecer o comportamento das áreas de vegetação remanescentes no bioma em relação a este serviço ambiental. O reconhecimento de padrões característicos da vegetação e o levantamento de variáveis e informações indispensáveis às práticas de manejo sustentável, são imprescindíveis para alcançar medidas conservacionistas para o bioma altamente ameaçado.

Deste modo, é relevante o desenvolvimento de estudos visando à obtenção de estimativas adequadas do estoque de carbono nas diferentes regiões e fitofisionomias que compõem o Cerrado, para a inclusão destas em modelos de manejo florestal apropriados para o bioma (REZENDE et al., 2006; RIBEIRO et al., 2011). Conforme Paiva, Rezende e Pereira (2011), a falta de estudos relacionados à quantificação da biomassa total no Cerrado compromete o entendimento do processo de conversão do gás carbônico atmosféricos no bioma. Para as formações savânicas, aponta-se que tais estimativas adequadas são escassas, principalmente devido à grande diversidade de espécies, à alta variabilidade existente entre indivíduos de uma mesma espécie, além da grande variação na forma do tronco e copa dos indivíduos (REZENDE et al., 2006).

Concomitante à natural variabilidade intrínseca das árvores de qualquer área de vegetação savânica de Cerrado, está também a variação na composição e estrutura em áreas de cerrados sensu stricto que se desenvolvem sobre diferentes tipos de solos (FELFILI et al., 2004). No entanto, a literatura mostra que a maioria dos estudos que visam quantificar biomassa e carbono nestes ambientes concentram-se em áreas no Distrito Federal e em Minas Gerais, referindo-se na maior parte a cerrados desenvolvidos sobre solos mais argilosos (REZENDE et al., 2006; PAIVA; REZENDE; PEREIRA, 2011; RIBEIRO et al., 2011). Para outras fitofisionomias e áreas de cerrado sensu stricto no oeste baiano, hoje intensamente pressionadas pela mudança no uso do solo, são escassos os trabalhos desta natureza. Neste sentido, este estudo teve o objetivo de ajustar e comparar modelos estatísticos de predição da biomassa individual de árvores e estimar os estoques totais de biomassa seca e carbono acima do solo nos três compartimentos (arbóreo, herbáceo-arbustivo e serapilheira) de um cerrado típico localizado em área de influência do Arenito Urucuia, no oeste da Bahia.

\section{Material e métodos}

\section{Área de estudo}

O estudo foi desenvolvido em uma área contínua do bioma Cerrado de aproximadamente 9.570 ha, localizada no município de Jaborandi, oeste baiano, entre as coordenadas $14^{\circ} 42^{\prime}$ S e $45^{\circ} 53^{\prime}$ N. A altitude varia entre $765 \mathrm{~m}$ e $943 \mathrm{~m}$, e o relevo é predominantemente suave ondulado. O clima regional conforme a classificação de Köppen, é Aw e caracterizado como Tropical Sazonal de Inverno Seco. A temperatura média anual é aproximadamente $24^{\circ} \mathrm{C}$, com mínima de $18^{\circ} \mathrm{C}$ e máxima de $34^{\circ} \mathrm{C}$ e a precipitação média anual varia de $700 \mathrm{~mm}$ a $1.400 \mathrm{~mm}$, sendo os meses de novembro, dezembro, janeiro e março os de maior precipitação. 
A área deste contínuo de Cerrado insere-se na região de ocorrência do imenso chapadão que constitui a Formação Urucuia localizado a oeste da média bacia do São Francisco (CASTRO et al., 2010). Neste substrato litológico, predominam arenitos quartzosos, cujos solos são predominantemente de textura franco-arenosa, com domínio de Latossolos e Neossolos Quartzarênicos (SILVA et al., 2015). A área está totalmente inserida no Sistema Aquífero Urucuia, sendo quase inteiramente coberta por vegetação nativa do Cerrado, com grande diversidade de ambientes. O cerrado típico é a fitofisionomia mais abundante, e se estende por aproximadamente $64 \%$ da área total deste contínuo do bioma. $\mathrm{Na}$ área também estão presentes o cerrado ralo, o cerrado denso, os campos limpos e campos sujos, as veredas, as matas de galeria e o cerradão distrófico (SILVA et al., 2015).

O estudo foi conduzido em uma área menor de 30 ha inteiramente coberta por vegetação de cerrado típico, o que representa $0,5 \%$ da área total. A área foi definida em função da representatividade real da fitofisionomia e por não estar designada legalmente como unidade de conservação, reserva legal ou área de preservação permanente de qualquer natureza, tornando possível a obtenção de autorização do órgão ambiental competente para supressão das árvores, que foram utilizadas para a amostragem destrutiva de quantificação da biomassa. Nesta área de 30 ha, primeiramente foi realizado o inventário florestal para conhecimento da composição florística e da estrutura do componente arbóreo do cerrado típico.

Para isso, foram alocadas aleatoriamente 20 parcelas de $1.000 \mathrm{~m}^{2}(20 \mathrm{~m} \times 50 \mathrm{~m})$ na área de pesquisa. Em cada uma das parcelas foram coletados os dados de diâmetro e altura total das árvores que atenderam ao critério de inclusão do diâmetro medido a $30 \mathrm{~cm}$ do solo $\left(\mathrm{d}_{30 \mathrm{~cm}}\right)$ igual ou superior a $5 \mathrm{~cm}$ $\left(\mathrm{d}_{30 \mathrm{~cm}} \geq 5 \mathrm{~cm}\right)$. As árvores mensuradas tiveram o material botânico coletado para identificação das espécies botânicas presentes.

\section{Amostragem dos compartimentos arbóreo, herbáceo-arbustivo e serapilheira}

Para o ajuste dos modelos estatísticos de predição da biomassa de árvores (compartimento arbóreo), foram amostradas 90 árvores distribuídas em diferentes classes diamétricas e espécies, conforme estrutura e composição de espécies do cerrado típico estudado, descritas em Oliveira et al. (2015).

Ao serem localizadas em campo, o procedimento para coleta de dados de cada uma das 90 árvores procedeu-se da seguinte maneira: primeiramente a árvore foi mensurada, determinando-se o diâmetro a 30 $\mathrm{cm}$ do solo $\left(\mathrm{d}_{30 \mathrm{~cm}}\right)$ e a altura do peito $\left(\right.$ dap $\left._{1,30 \mathrm{~m}}\right)$. Depois de mensurada, a árvore foi cortada a $30 \mathrm{~cm}$ do solo sobre uma lona resistente, e teve a altura total mensurada com trena. Tomadas as medidas dendrométricas, iniciaram-se os procedimentos de separação dos componentes da árvore, começando pelos mais delicados, que são as flores, seguidos pelos frutos, folhas, galhos finos, galhos grossos e tronco. Os componentes separados foram pesados em balanças de diferentes capacidades $(1 \mathrm{~kg}, 5 \mathrm{~kg}, 20 \mathrm{~kg}, 45 \mathrm{~kg}, 50 \mathrm{~kg}$ e 100 $\mathrm{kg}$ ) com auxílio de sacos e cordas. Ressalta-se que neste trabalho a biomassa foi obtida para a árvore inteira, considerando todos os componentes presentes. Depois de pesar todo o material úmido, de todos os componentes separadamente da árvore, amostras de cada componente foram coletadas e acondicionadas em sacos plásticos com a devida identificação da árvore. Essas amostras foram levadas ao laboratório para obtenção do teor de umidade e peso de matéria seca. As amostras dos troncos foram obtidas a partir de discos retirados nas posições $0,30 \mathrm{~m}$, na metade da altura total e na altura total (ponta).

Para a amostragem do compartimento herbáceo-arbustivo foram considerados os indivíduos lenhosos arbustivos com $\mathrm{d}_{30 \mathrm{~cm}}<5 \mathrm{~cm}$ e ht $\leq 1 \mathrm{~m}$, e a vegetação rasteira, incluindo pequenas ervas e gramíneas. A amostragem para quantificação da biomassa neste compartimento foi realizada por meio de 20 subparcelas fixas de $4 \mathrm{~m}^{2}(2 \mathrm{~m} \times 2 \mathrm{~m})$. As subparcelas de $4 \mathrm{~m}^{2}$ foram alocadas sistematicamente no centro das parcelas maiores do inventário florestal $(20 \mathrm{~m}$ x $50 \mathrm{~m})$. Em cada uma das 20 subparcelas, todo o material úmido presente foi separado nos componentes: folhas, gravetos e gramíneas, e pesado separadamente. Amostras de cada componente foram tomadas para obtenção dos teores de umidade e peso de matéria seca em laboratório.

A amostragem da biomassa presente na serapilheira foi realizada por 20 subparcelas fixas de $1 \mathrm{~m}^{2}$ $(1 \mathrm{~m} \mathrm{x} 1 \mathrm{~m})$. Estas subparcelas também foram alocadas sistematicamente dentro das parcelas maiores do inventário florestal. O procedimento foi de alocação da subparcela em um dos quatro cantos da parcela 
maior do inventário florestal, permitindo-se a variação do canto escolhido em cada parcela. Optou-se por variar o canto de alocação da subparcela, para tentar amostrar melhor a heterogeneidade de distribuição da serapilheira no ambiente, que ora ocorre escassa nas áreas abertas da paisagem e ora concentrada sob as árvores. Em cada uma das 20 subparcelas, todo o material úmido presente foi pesado e uma amostra foi coletada para obtenção do teor de umidade e peso de matéria seca em laboratório.

No laboratório, as amostras úmidas foram pesadas em balança de precisão e posteriormente secas em estufa de circulação forçada de ar, a uma temperatura em torno de $70^{\circ} \mathrm{C}$ até a obtenção de massa constante.

\section{Estimativa do compartimento arbóreo}

A partir dos dados obtidos de cada árvore pesada, foram ajustados modelos estatísticos para a estimativa da biomassa seca por árvore de forma indireta (não destrutiva), em função das variáveis diâmetro $\left(\mathrm{d}_{30 \mathrm{~cm}}\right.$ ou dap $\left.\mathrm{p}_{1,30 \mathrm{~m}}\right)$ e altura total (ht), bem como suas combinações. Para tanto, foram testados diferentes modelos lineares e não lineares conforme Scolforo, Oliveira e Acerbi Júnior (2008) (Tabela 1).

Os modelos lineares foram ajustados por análise de regressão, utilizando o método dos mínimos quadrados ordinários para a estimativa dos parâmetros. Os modelos não lineares foram ajustados por iterações. Os dados foram testados quanto aos pressupostos de normalidade e homogeneidade, por meio dos testes de Shapiro-Wilk $(\alpha=0,05)$ e teste $\mathrm{f}(\alpha=0,05)$. Foi feita a análise gráfica para verificação da correlação entre as variáveis dependente e independentes. A escolha do melhor modelo baseou-se no coeficiente de determinação ajustado $\left(\mathrm{R}_{\text {ajust. }}^{2}\right)$, na significância dos coeficientes a $95 \%$ de probabilidade, no erro padrão de estimativa em termos percentuais $\left(\mathrm{S}_{x y} \%\right)$ e na distribuição gráfica dos resíduos. Para os modelos com variável dependente transformada, o erro padrão residual foi corrigido na escala original da variável dependente, conforme Scolforo (2005). A análise da diferença entre os valores observados e os estimados pela equação escolhida foi realizada pelo teste t pareado $(\alpha=0,05)$.

Tabela 1 - Modelos estatísticos testados para estimar os estoques de biomassa seca das árvores.

Table 1 - Statistical models tested to estimate dry biomass stocks of trees.

\begin{tabular}{|c|c|}
\hline Autor & Modelo \\
\hline Hohenald - Krenm & $y=\beta_{0+} \beta_{1}(d)+\beta_{2}\left(d^{2}\right)+\varepsilon$ \\
\hline Brenac & $\ln (\mathrm{y})=\beta_{0+} \beta_{1} \ln (\mathrm{d})+\beta_{2}(1 / \mathrm{d})+\ln \varepsilon$ \\
\hline Spurr & $y=\beta_{0}+\beta_{1} d^{2} h+\varepsilon$ \\
\hline Schumacher \& Hall & $y=\beta_{0} d^{\beta 1} h^{\beta 2}+\varepsilon$ \\
\hline Schumacher \& Hall (logarítmica) & $\begin{array}{c}\ln (\mathrm{y})=\beta_{0+} \beta_{1} \ln (\mathrm{d})+\beta_{2} \ln (\mathrm{h})+\ln \varepsilon \\
\mathrm{y}=\quad \mathrm{d}^{2}+\varepsilon\end{array}$ \\
\hline Honner & $\begin{array}{r}\beta_{0}+\beta_{1} 1 \\
\mathrm{H}\end{array}$ \\
\hline Ogaya & $y=d^{2}\left(\beta_{0}+\beta_{1} h\right)+\varepsilon$ \\
\hline Stoate & $y=\beta_{0}+\beta_{1} d^{2}+\beta_{2} d^{2} h+\beta_{3} h+\varepsilon$ \\
\hline Naslund & $y=\beta_{1} d^{2}+\beta_{2} d^{2} h+\beta_{3} d h^{2}+\beta_{4} h^{2}+\varepsilon$ \\
\hline Takata & $\mathrm{y}=\underline{\mathrm{d}} \underline{\underline{2}} \underline{\beta_{0}+\beta_{1} \mathrm{~d}}+\varepsilon$ \\
\hline Spurr (logarítmica) & Lny $=\beta_{0}+\beta_{1} \operatorname{Ln}\left(d^{2} h\right)+\varepsilon$ \\
\hline Meyer & $y=\beta_{0}+\beta_{1} d+\beta_{2} d^{2}+\beta_{3} d h+\beta_{4} d^{2} h+\beta_{5} h+\varepsilon$ \\
\hline
\end{tabular}

Em que: $\mathrm{y}=$ Biomassa seca $(\mathrm{kg}) ; \mathrm{d}=\mathrm{d}_{30 \mathrm{~cm}} / \mathrm{dap}_{1,30 \mathrm{~m}} ; \mathrm{h}=$ altura total. 
Estimativa dos estoques de biomassa seca e carbono

Para os compartimentos herbáceo-arbustivo e serapilheira, os valores de biomassa úmida obtidos em campo foram convertidos em quantidade de biomassa seca por hectare a partir dos teores de umidade determinados em laboratório. Para estimar o estoque de biomassa seca do compartimento arbóreo, a equação alométrica escolhida foi aplicada aos dados levantados no inventário florestal. Isso permitiu estimar os estoques tanto por hectare, quanto por indivíduos, espécies e classes diamétricas do compartimento arbóreo do cerrado típico estudado. As estimativas para cada compartimento foram feitas por intervalo de confiança a $95 \%$ de probabilidade $(\infty=0,05)$. Para os compartimentos arbóreo e herbáceo-arbustivo, a biomassa seca total foi convertida em carbono considerando a proporção de 47\% (INTERGOVERNMENTAL PANEL ON CLIMATE CHANGE, 2006). Para a serapilheira, a concentração de carbono foi considerada 44,36\% (MORAIS, 2012).

\section{Resultados e Discussão}

Ajuste dos modelos para estimativa do estoque de biomassa seca

A amostra para determinação da biomassa seca no compartimento arbóreo do cerrado típico contou com 90 árvores distribuídas em seis classes diamétricas (Tabela 2) e entre 15 espécies botânicas diferentes, conforme os dados levantados no inventário florestal. No inventário florestal foram levantadas 57 espécies lenhosas de 41 gêneros e 23 famílias botânicas. A densidade total foi estimada em 951 ind. ha ${ }^{-1}$ com área basal de $7,85 \mathrm{~m}^{2} \mathrm{ha}^{-1}$. O erro amostral do inventário florestal para a estimativa destas variáveis foi respectivamente de $7 \%$ e $9 \%(\infty=0,05)$. As 15 espécies escolhidas para compor a amostra de árvores para determinação da biomassa representam $26 \%$ do total de espécies presentes na área. Estas espécies foram escolhidas em função do valor de importância (VI). Foram amostradas principalmente espécies de maiores VI, bem como outras de menores VI, visando garantir uma amostra representativa das principais espécies do ambiente.

\section{Tabela 2 - Frequência de árvores amostradas por classe diamétrica $(\mathrm{cm})$ para quantificação da biomassa por método destrutivo.}

Table 2 - Frequency of arboreal individuals sampled by diameter class $(\mathrm{cm})$ for the quantification of biomass by a destructive method.

\begin{tabular}{lcc}
\hline Classes de $\mathbf{d}_{\mathbf{3 0 \mathrm { cm }}}$ & Classes de dap $_{\mathbf{1 , 3 0 \mathrm { m }}}$ & Número de árvores amostradas \\
\hline $5,0-8,9$ & $3,5-8,5$ & 23 \\
$9,0-12,9$ & $8,5-13,5$ & 22 \\
$13,0-16,9$ & $13,5-17,5$ & 17 \\
$17,0-20,9$ & $17,5-22,5$ & 15 \\
$21,0-24,9$ & $22,5-27,5$ & 7 \\
$>24,9$ & $>27,5$ & 6 \\
\hline Total & Total & 90 \\
\hline
\end{tabular}

Todos as equações ajustadas para a estimativa da biomassa em função do $d_{30 \mathrm{~cm}}(\mathrm{~cm})$ foram significativas $(p<0,05)$. As variáveis dependentes e independentes apresentaram correlação positiva. O coeficiente de determinação ajustado $\left(\mathrm{R}^{2}{ }_{\text {ajust. }}\right.$ ), variou de $87,76 \%$ a $94,10 \%$ mostrando alta correlação existente entre a variável dependente (biomassa) e variáveis independentes $\left(\mathrm{d}_{30 \mathrm{~cm}}\right.$ e ht). Os erros padrões percentuais variaram de 49,12\% a 55,61\% (Tabela 3). Os elevados erros decorrem da grande diversificação na forma dos troncos e copa típica das árvores do Cerrado. As características dendrométricas, bem como os respectivos estoques por componente das árvores que foram mensuradas e pesadas para o ajuste dos modelos, são apresentadas na Tabela 4, em que se observa grande variação na biomassa de todos os componentes das árvores . Cabe ressaltar que neste trabalho, as equações foram ajustadas para estimar o estoque de 
biomassa seca de toda a árvore, ou seja, considera a presença de folhas, galhos finos, galhos grossos, tronco, flores e frutos. Isso eleva a variabilidade dos dados amostrados, devido à grande heterogeneidade na forma das copas das árvores amostradas. Conforme Scolforo, Oliveira e Acerbi Júnior (2008), elevados valores dos erros padrões percentuais decorrem da alta heterogeneidade da amostra, típica de vegetações nativas, sobretudo do Cerrado, onde a variação das copas é muito grande.

Tabela 3 - Estimativa dos parâmetros e medidas de precisão das equações ajustadas para a estimativa do estoque em biomassa seca $(\mathrm{kg})$ em função do $d_{30 \mathrm{~cm}}(\mathrm{~cm})$ e/ou ht $(\mathrm{m})$.

Table 3 - Estimation of the parameters and precision measurements of the equations adjusted for estimating the stocks in dry biomass $(\mathrm{kg})$ as a function of $\mathrm{d}_{30 \mathrm{~cm}}(\mathrm{~cm})$ and/or $\mathrm{ht}(\mathrm{m})$.

\begin{tabular}{|c|c|c|c|c|c|c|c|c|c|}
\hline Modelo & $\beta 0$ & $\beta 1$ & $\beta 2$ & $\beta 3$ & $\beta 4$ & $\beta 5$ & $\begin{array}{c}\mathbf{R 2 \%} \\
\text { (ajust.) }\end{array}$ & Sxy (kg) & Sxy (\%) \\
\hline Hohenald Krenm & 18,434 & -49.134 & 0,392 & - & - & - & 89,14 & 22,4 & 49,36 \\
\hline Brenac & $-3,378$ & 2,617 & $-4,3261 *$ & - & - & - & 93,1 & 22,89 & 50,41 \\
\hline Spurr & $-1,364$ & 0,036 & - & - & - & - & 87,79 & 23,76 & 52,33 \\
\hline $\begin{array}{l}\text { Schumacher \& Hall } \\
\text { (logarítmica) }\end{array}$ & $-4,439$ & 2,420 & 0,865 & - & - & - & 94,1 & 24,93 & 54,91 \\
\hline Stoate (australiana) & $-1,328$ & $0,1328 *$ & 0,020 & $-2,8213^{*}$ & - & - & 88,55 & 23,01 & 50,68 \\
\hline Naslund & $-11,144$ & $0,0457 *$ & $0,0553^{*}$ & $-0,1523 *$ & $1,3728^{*}$ & - & 88,77 & 22,78 & 50,18 \\
\hline Spurr (logarítmica) & $-4,320$ & 1,119 & - & - & - & - & 94,07 & 25,25 & 55,61 \\
\hline Meyer & 38,892 & $-11,2766^{*}$ & $0,5905^{*}$ & $0,6952 *$ & $-0,0263^{*}$ & $1,9967^{*}$ & 89,24 & 22,3 & 49,12 \\
\hline Honner & 1,237 & 19,922 & - & - & - & - & 88,29 & 23,26 & 51,23 \\
\hline Takata & 27,847 & 0,014 & - & - & - & - & 87,76 & 23,78 & 52,38 \\
\hline Ogaya & 0,045 & 0,028 & - & - & - & - & 88,02 & 23,53 & 51,83 \\
\hline Schumacher \& Hall & 0,029 & 2,528 & 0,174 & - & - & - & 88,98 & 22,57 & 49,71 \\
\hline
\end{tabular}

* Coeficientes não significativos $(\mathrm{p}>0,05)$

Tabela 4 - Valores mínimos e máximos, média e desvio padrão, relativos às características dendrométricas e diferentes estoques por componentes das árvores amostradas para o ajuste dos modelos estatísticos.

Table 4 - Minimum and maximum values, mean and standard deviation, relative to the dendrometric characteristics and different stocks per components of the trees sampled for the adjustment of the statistical models.

\begin{tabular}{lcccc}
\hline Variáveis & Máximo & Mínimo & Média & Desvio padrão \\
\hline $\mathrm{d}_{30 \mathrm{~cm}}(\mathrm{~cm})$ & 36,45 & 5,09 & 14,11 & 6,86 \\
$\mathrm{dap}_{1,30 \mathrm{~m}}(\mathrm{~cm})$ & 35,01 & 3,5 & 12,45 & 6,93 \\
$\mathrm{ht}(\mathrm{m})$ & 8,29 & 1,8 & 4,15 & 1,49 \\
Peso - folhas $(\mathrm{kg})$ & 19,84 & 0,035 & 3,12 & 4,4 \\
Peso - galhos finos $(\mathrm{kg})$ & 58,32 & 0,17 & 9,02 & 12,95 \\
Peso - galhos grossos $(\mathrm{kg})$ & 133,4 & 0,212 & 18,66 & 26,67 \\
Peso - tronco $(\mathrm{kg})$ & 469,16 & 1,031 & 45,54 & 71,1 \\
Peso - frutos $(\mathrm{kg})$ & 8,03 & 0,042 & 1,24 & 1,87 \\
Peso - flores $(\mathrm{kg})$ & 2,2 & 0,04 & 0,624 & 0,89 \\
Peso - total $(\mathrm{kg})$ & 557,84 & 1,548 & 76,27 & 107,15 \\
Biomassa seca - total $(\mathrm{kg})$ & 415,73 & 0,784 & 45,4 & 68 \\
Carbono $(\mathrm{kg})$ & 207,87 & 0,392 & 22,7 & 34 \\
\hline
\end{tabular}


As equações ajustadas a partir dos modelos de Schumacher e Hall (logarítmica) e de Spurr (logarítmica) apresentaram valores semelhantes e os mais elevados para o coeficiente de determinação ajustado $\left(\mathrm{R}_{\text {ajust. }}^{2}\right.$ ), em torno de $94,1 \%$, bem como os maiores erros padrões percentuais, $54,91 \%$ e $55,61 \%$, respectivamente. Embora tenham apresentado erros percentuais mais elevados que as demais equações ajustadas, a distribuição gráfica dos resíduos destas equações mostrou que elas ofereceram melhores estimativas da variável dependente, fornecendo estimativas livres de tendenciosidades (Figura 1).

\section{Figura 1 - Distribuição dos resíduos para os modelos ajustados em função do $d_{30 \mathrm{~cm}}(\mathrm{~cm})$ e ht $(\mathrm{m})$.}

Figure 1 - Distribution of the residuals for the adjusted models according to the $\mathrm{d}_{30 \mathrm{~cm}}(\mathrm{~cm})$ and $\mathrm{ht}(\mathrm{m})$.
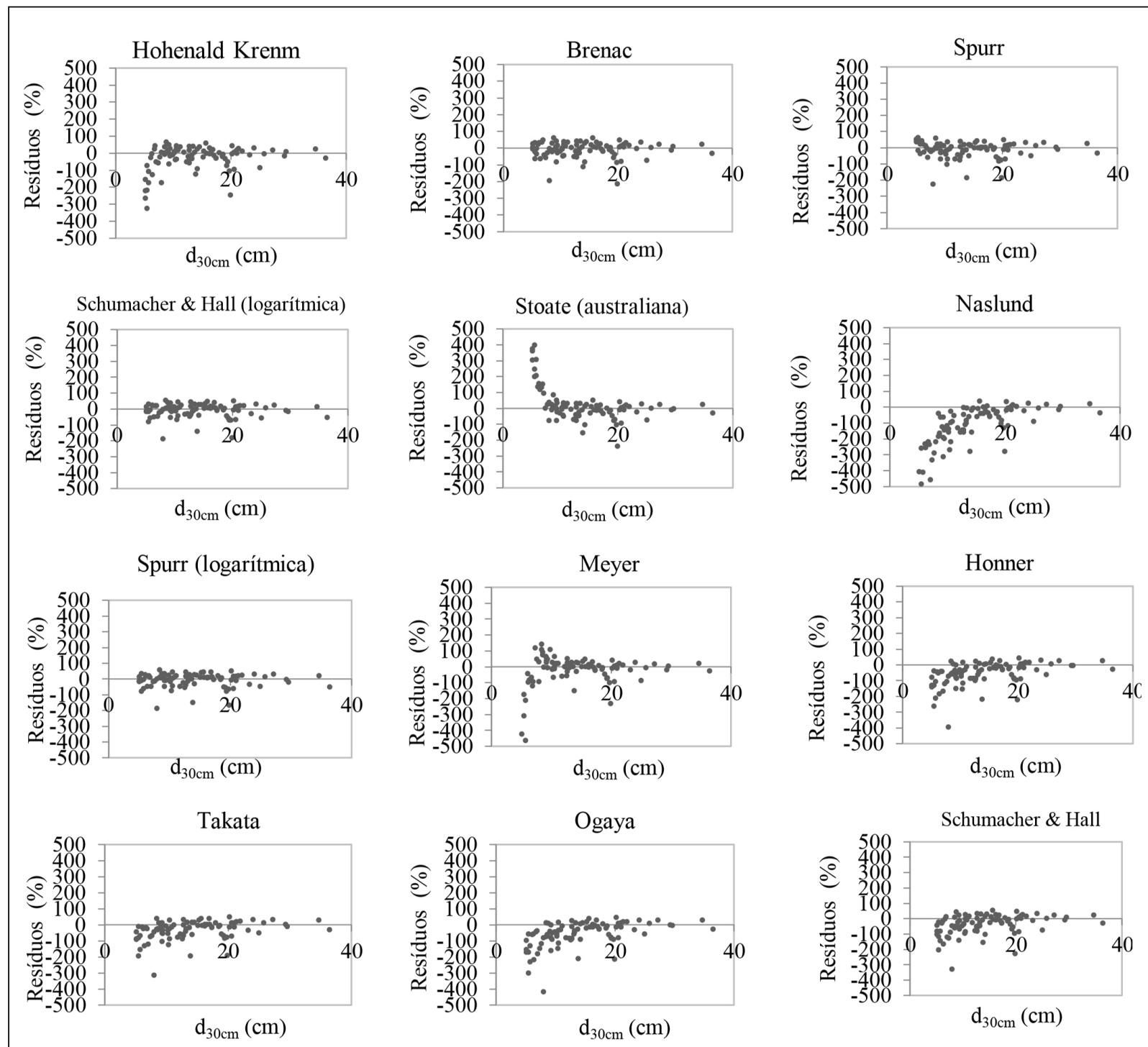

A equação ajustada do modelo de Brenac também apresentou bons resultados, com coeficiente de determinação de $93,10 \%$, erro padrão de $50,41 \%$ e distribuição gráfica dos resíduos indicando estimativas livres de tendências. Esses resultados revelam que a equação ajustada, proveniente deste modelo, é capaz de produzir boas estimativas tendo apenas o diâmetro como variável preditora, podendo ser utilizada caso dados de altura não sejam coletados no inventário florestal. Os demais modelos apresentaram $\mathrm{R}_{\text {ajust. }}^{2}$ inferiores a $90 \%$ e tendenciosidades na distribuição gráfica dos resíduos, indicando ineficiência nas estimativas. Deste modo, com base nas medidas de precisão, bem como na dispersão gráfica dos resíduos, a equação ajustada proveniente do modelo de Schumacher \& Hall (logarítmica) foi a escolhida para 
estimar os estoques individuais de biomassa seca das árvores no cerrado típico aqui estudado. Não houve diferença significativa entre os valores observados e os estimados pela equação, quando comparados pelo teste t pareado $(\alpha=0,05)$.

$$
\text { Biomassa seca (kg): In (bs) }=-4,4389+2,4199 \operatorname{In}(\mathrm{d})+0,8650 \mathrm{In}(\mathrm{ht})
$$

Em que: bs $=$ Biomassa seca $(\mathrm{kg}) ; \mathrm{d}=\mathrm{d}_{30 \mathrm{~cm}}(\mathrm{~cm}) ; \mathrm{ht}=$ Altura total $(\mathrm{m})$

Todos as equações ajustadas em função do dap ${ }_{1,30 \mathrm{~m}}(\mathrm{~cm})$ foram significativas $(p<0,05)$. Os valores de $\mathrm{R}_{\text {ajust. }}^{2}$ variaram de $91,84 \%$ a $93,58 \%$ e os erros padrões percentuais entre $40,64 \%$ e $46,12 \%$ (Tabela 5). Observa-se que as medidas de precisão variaram pouco entre os modelos ajustados. Deste modo, combinando as medidas de precisão com a distribuição gráfica dos resíduos (Figura 2), concluiu-se que a equação proveniente do ajuste do modelo de Schumacher \& Hall foi capaz de proporcionar as melhores estimativas de biomassa seca quando se utilizou o dap $_{1,30 \mathrm{~m}}$ e a altura total como variáveis preditoras. Não houve diferença significativa entre os valores observados e estimados pela equação, quando comparados pelo teste $t$ pareado $(\alpha=0,05)$. Destaca-se que, considerando o dap $_{1,30 \mathrm{~m}}(\mathrm{~cm})$, as equações ajustadas neste trabalho são capazes de garantir boas estimativas da variável dependente considerando uma variação diamétrica de $3,5 \mathrm{~cm}$ a $35,0 \mathrm{~cm}$ para o diâmetro medido a $1,30 \mathrm{~m}$ do solo, visto que esta foi a variação diamétrica contemplada na amostra para ajuste dos modelos em relação ao dap 1,30m $_{1}(\mathrm{~cm})$.

$$
\text { Biomassa seca }(\mathrm{kg}): \mathrm{bs}=0,0441 \mathrm{dap}^{2,2872} \mathrm{ht}^{0,4606}
$$

Em que: $\mathrm{bs}=$ Biomassa seca $(\mathrm{kg}) ;$ dap $=$ Diâmetro a 1,30 $\mathrm{m}$ do solo $(\mathrm{cm}) ; \mathrm{ht}=$ Altura total $(\mathrm{m})$.

Tabela 5 - Estimativa dos parâmetros e medidas de precisão das equações ajustadas para a estimativa do estoque de biomassa seca em função do dap ${ }_{1,30 \mathrm{~cm}}(\mathrm{~cm})$ e ht $(\mathrm{m})$.

Table 5 -Estimation of the parameters and precision measurements of the adjusted equations for the estimation of the dry biomass stock as a function of dap $p_{1,30 \mathrm{~cm}}(\mathrm{~cm})$ and $\mathrm{ht}(\mathrm{m})$.

\begin{tabular}{lccccccccc}
\hline Modelo & $\boldsymbol{\beta 0}$ & $\boldsymbol{\beta 1}$ & $\boldsymbol{\beta 2}$ & $\boldsymbol{\beta 3}$ & $\boldsymbol{\beta 4}$ & $\boldsymbol{\beta 5}$ & $\mathbf{R 2} \%$ (ajust.) & $\mathbf{S x y}(\mathbf{k g})$ & $\mathbf{S x y}(\mathbf{\%})$ \\
\hline Hohenald Krenm & 14,305 & $-4,104$ & 0,406 & - & - & - & 91,84 & 19,43 & 42,79 \\
Brenac & $-3,684$ & 2,692 & $1,6366^{*}$ & - & - & - & 92,55 & 19,39 & 42,72 \\
Spurr & 0,550 & 0,041 & - & - & - & - & 91,92 & 19,33 & 42,57 \\
Schumacher \& Hall & $-3,086$ & 2,027 & 0,852 & - & - & - & 93,53 & 20,08 & 44,92 \\
(logaritmica) & & & & & & & & & \\
Stoate (australiana) & 3,135 & $0,0985^{*}$ & 0,029 & $-2,2380^{*}$ & - & - & 92,25 & 18,93 & 41,69 \\
Naslund & $-6,047$ & $0,0138^{*}$ & 0,063 & $-0,1366^{*}$ & $1,2182^{*}$ & - & 92,52 & 18,6 & 40,96 \\
Spurr (logarítmica) & $-3,078$ & 0,976 & - & - & - & - & 93,58 & 20,36 & 46,12 \\
Meyer & $-1,176$ & $-2,9743^{*}$ & $0,2670^{*}$ & $-0,2671^{*}$ & $0,0196^{*}$ & $5,7833^{*}$ & 92,34 & 18,81 & 41,44 \\
Honner & 1,032 & 17,503 & - & - & - & - & 92,32 & 18,83 & 41,48 \\
Takata & 25,443 & $-0,037$ & - & - & - & - & 91,92 & 19,32 & 42,55 \\
Ogaya & 0,057 & 0,032 & - & - & - & - & 92,19 & 19 & 41,85 \\
Schumacher \& Hall & 0,044 & 2,287 & 0,461 & - & - & - & 92,63 & 18,45 & 40,64 \\
\hline
\end{tabular}

* Coeficientes não significativos $(\mathrm{p}>0,05)$ 
Figura 2 - Distribuição dos resíduos para os modelos ajustados em função do $d_{1,30 \mathrm{~m}} \mathrm{e}$ ht (m).

Figure 2 - Distribution of the residuals for the adjusted models according to $\mathrm{d}_{1,30 \mathrm{~m}}$ and $\mathrm{ht}(\mathrm{m})$.
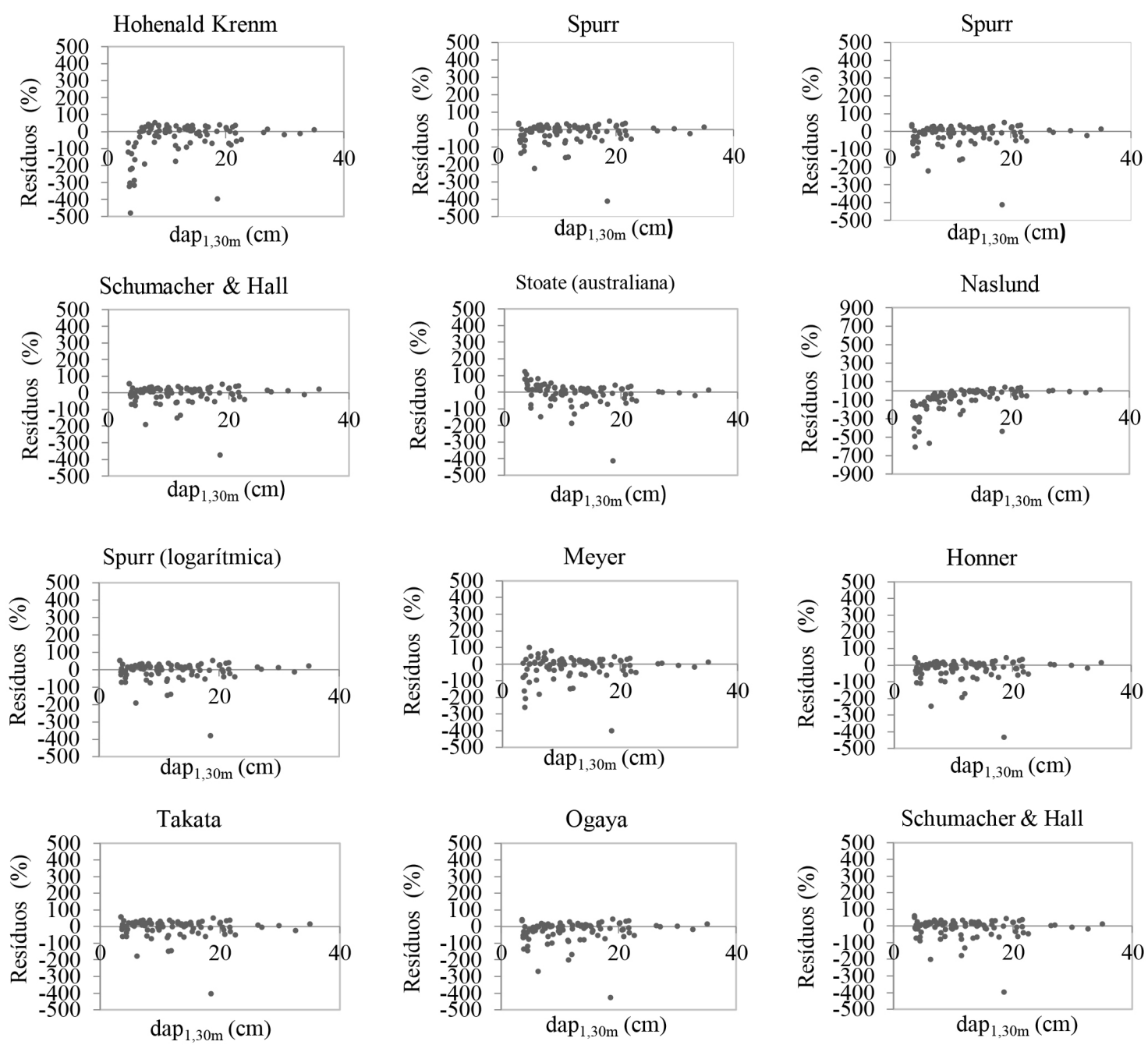

\section{Estoques de biomassa e carbono no compartimento arbóreo}

O compartimento arbóreo do cerrado típico tem em média 10,9 $\mathrm{Mg} \mathrm{ha}^{-1}$ de biomassa seca estocada, o que corresponde ao estoque médio de $5,1 \mathrm{Mg} \mathrm{ha}^{-1} \mathrm{de}$ carbono neste compartimento. (Tabela 6).

Tabela 6 - Estoque de biomassa seca e carbono, densidade e dominância de indivíduos no compartimento arbóreo.

Table 6 -Stocks in dry biomass and carbon, density and dominance of individuals in the woody compartment.

\begin{tabular}{lccccc}
\hline Variável & Média & $\mathbf{S}_{\overline{\mathbf{y}}} \mathbf{t}$ & $\left(\mathbf{S}_{\mathbf{y}} \mathbf{t}\right) \%$ & Limite inferior & Limite superior \\
\hline Densidade (ind. ha-1) & 952 & 66 & 6,96 & 885 & 1.018 \\
Área basal $\left(\mathrm{m}^{2} \mathrm{ha}^{-1}\right)$ & 7,85 & 0,74 & 9,32 & 7,12 & 8,59 \\
Biomassa $\left(\mathrm{Mg} \mathrm{ha}^{-1}\right)$ & 10,9 & 1,7 & 15,84 & 9,2 & 12,6 \\
Carbono $\left(\mathrm{Mg} \mathrm{ha}^{-1}\right)$ & 5,1 & 0,8 & 15,84 & 4,3 & 5,9 \\
\hline
\end{tabular}

Em que: $\mathrm{S}_{\bar{y}} \mathrm{t}=$ Erro amostral em termos absolutos; $\left(\mathrm{S}_{\bar{y}} \mathrm{t}\right) \%=$ Erro amostral em termos relativos. $(\alpha=0,05)$ 
Os valores dos estoques de biomassa e carbono verificados para o compartimento arbóreo do cerrado típico estudado estão dentro do intervalo observado para outras áreas de cerrado sensu stricto e sugerem que os estoques em cerrados sobre solos oriundos do arenito Urucuia se encontram no limite inferior desse intervalo. Em 25 localidades de cerrado sensu stricto estudadas por Felfili (2008), o estoque de carbono no compartimento arbóreo variou de 3,72 $\mathrm{Mg} \mathrm{ha}^{-1}$ a 13,27 $\mathrm{Mg} \mathrm{ha}^{-1}$, sendo que na região do Espigão Mestre do São Francisco, os valores variaram de 5,33 $\mathrm{Mg} \mathrm{ha}^{-1}$ a 9,16 $\mathrm{Mg} \mathrm{ha}^{-1}$. Para duas áreas amostradas sobre Neossolos Quartzarênicos nos municípios de Correntina e São Desidério, ambos no oeste da Bahia, foram verificados valores de 5,26 Mg ha- e 5,33 $\mathrm{Mg} \mathrm{ha}^{-1}$ respectivamente, corroborando os resultados verificados para a área do presente estudo. Ottmar et al. (2001) observaram estoque de biomassa do compartimento arbóreo variando de 12,53 $\mathrm{Mg} \mathrm{ha}^{-1}$ a 42,96 Mg ha-1 para cinco diferentes localidades de cerrado sensu stricto. Sustentando o presente resultado encontrado para o cerrado típico, o menor valor reportado pelos autores $\left(12,53 \mathrm{Mg} \mathrm{ha}^{-1}\right)$ refere-se ao cerrado sensu stricto amostrado em área do Parque Nacional Grande Sertão Veredas, também sobre solos arenosos na região do Espigão Mestre do São Francisco.

Em relação à contribuição por espécies, foi observado que cinco delas foram responsáveis por estocar mais de $50 \%$ da biomassa e carbono presentes no compartimento arbóreo (Tabela 7). Dessas espécies, quatro pertencem ao grupo das sete espécies com maiores valores de importância, sendo elas: Pouteria ramiflora, Salvertia convallariodora, Kielmeyera coriacea e Pouteria torta. As espécies Hymenaea stigonocarpa, Myrocarpus fastigiatus, Bowdichia virgiloides e Qualea parviflora apresentaram expressivos estoques devido principalmente à presença de indivíduos nas classes diamétricas superiores. Conforme Haridasam (2005), apesar da elevada diversidade de indivíduos arbóreos em cerrado sensu stricto, um número pequeno de espécies constitui as populações mais densas e contribui para maiores estoques de biomassa e nutrientes. O autor observou ainda que do total de 35 espécies arbóreas, cinco foram responsáveis por $78 \%$ da biomassa total. Estas espécies apresentaram as menores concentrações de nutrientes foliares, indicando que a menor exigência de nutrientes parece conferir vantagem competitiva para estas espécies mais abundantes.

Ao relacionar o estoque de biomassa e carbono com a estrutura diamétrica do compartimento arbóreo no cerrado típico, observa-se acréscimo da primeira para a segunda classe, a partir da qual ocorre decréscimo até a penúltima classe. Sendo que, as três primeiras classes respondem por mais de $60 \%$ da biomassa e carbono estocados no componente arbóreo da fitofisionomia, com o máximo estoque concentrado na segunda classe diamétrica $\left(2,8 \mathrm{Mg} \mathrm{ha}^{-1}\right)$, que corresponde a cerca de $26 \%$ do estoque total de biomassa seca e carbono (Tabela 7). As duas primeiras classes diamétricas contemplam $100 \%$ das espécies encontradas no inventário florestal. Embora nas classes diamétricas superiores estejam os maiores estoques individuais, o elevado número de indivíduos nas menores classes faz com que os estoques por hectare sejam maiores neste intervalo, revelando que os indivíduos de pequeno porte constituem parte importante para manutenção dos estoques de biomassa e carbono neste ambiente.

Tabela 7 - Distribuição da biomassa seca $\left(\mathrm{Mg} \mathrm{ha}^{-1}\right)$ por espécies e classes diamétricas.

Table 7 - Distribution of dry biomass $\left(\mathrm{Mg} \mathrm{ha}^{-1}\right)$ by species and diameter classes.

\begin{tabular}{|c|c|c|c|c|c|c|c|c|c|}
\hline \multirow[t]{2}{*}{ Espécies } & \multicolumn{9}{|c|}{ Classes diamétricas $\mathrm{d} 30 \mathrm{~cm}(\mathrm{~cm})$} \\
\hline & 58,9 & $9-12,9$ & $13-16,9$ & 17-20,9 & $21-24,9$ & $25-28,9$ & 29-32,9 & $>33$ & $\sum$ \\
\hline $\begin{array}{l}\text { Pouteria ramiflora (Mart.) } \\
\text { Radlk* }\end{array}$ & 0,311 & 0,746 & 0,765 & 0,167 & 0,083 & 0,045 & - & 0,186 & 2,302 \\
\hline $\begin{array}{l}\text { Salvertia convallariaedora } \\
\text { A. St.-Hil.* }\end{array}$ & 0,018 & 0,027 & 0,189 & 0,177 & 0,26 & 0,16 & 0,171 & 0,439 & 1,442 \\
\hline $\begin{array}{l}\text { Kielmeyera coriacea } \\
\text { Mart. \& Zucc* }\end{array}$ & 0,253 & 0,547 & 0,195 & 0,087 & - & - & - & - & 1,082 \\
\hline $\begin{array}{l}\text { Pouteria torta (Mart.) } \\
\text { Radlk* }\end{array}$ & 0,165 & 0,252 & 0,193 & 0,147 & 0,042 & - & - & - & 0,799 \\
\hline
\end{tabular}


Tabela 7 - Continua...

Table 7-Continued...

\begin{tabular}{|c|c|c|c|c|c|c|c|c|c|}
\hline \multirow[t]{2}{*}{ Espécies } & \multicolumn{9}{|c|}{ Classes diamétricas $\mathrm{d} 30 \mathrm{~cm}(\mathrm{~cm})$} \\
\hline & 58,9 & $9-12,9$ & $13-16,9$ & $17-20,9$ & $21-24,9$ & $25-28,9$ & 29-32,9 & $>\mathbf{3 3}$ & $\sum$ \\
\hline $\begin{array}{l}\text { Hymenaea stigonocarpa } \\
\text { Mart. Ex Hayne }\end{array}$ & 0,034 & 0,091 & 0,041 & 0,103 & 0,109 & - & 0,083 & - & 0,461 \\
\hline $\begin{array}{l}\text { Myrocarpus fastigiatus } \\
\text { Allemão }\end{array}$ & 0,001 & 0,021 & 0,017 & 0,034 & 0,049 & - & - & 0,308 & 0,429 \\
\hline $\begin{array}{l}\text { Aspidospermas } \\
\text { tomentosum Mart.* }\end{array}$ & 0,174 & 0,131 & 0,053 & 0,017 & - & - & - & - & 0,375 \\
\hline $\begin{array}{l}\text { Bowdichia virgilioides } \\
\text { H.B.K. }\end{array}$ & 0,006 & - & 0,039 & - & 0,013 & 0,093 & - & 0,186 & 0,338 \\
\hline Qualea parviflora Mart. & 0,013 & 0,007 & 0,038 & - & 0,045 & 0,226 & - & - & 0,33 \\
\hline $\begin{array}{l}\text { Connarus suberosus } \\
\text { Planch.* }\end{array}$ & 0,084 & 0,15 & 0,062 & 0,025 & - & - & - & - & 0,321 \\
\hline $\begin{array}{l}\text { Ouratea hexasperma (St. } \\
\text { Hil.) Baill * }\end{array}$ & 0.037 & 0,106 & 0,127 & - & - & - & - & - & 0,27 \\
\hline $\begin{array}{l}\text { Caryocar brasilienses } \\
\text { Cambess. }\end{array}$ & 0,015 & 0,005 & 0,012 & 0,018 & - & 0,07 & 0,12 & - & 0,241 \\
\hline Macherium opacum Vog. & 0,007 & 0,043 & 0,034 & - & 0,131 & - & - & - & 0,215 \\
\hline $\begin{array}{l}\text { Andira vermífuga Mart. } \\
\text { ex Benth }\end{array}$ & 0,034 & 0,116 & 0,057 & - & - & - & - & - & 0,207 \\
\hline $\begin{array}{l}\text { Vochysea tucanorum } \\
\text { Mart. }\end{array}$ & 0,051 & 0,039 & 0,013 & 0,057 & 0,038 & - & - & - & 0,198 \\
\hline $\begin{array}{l}\text { Kyelmeyera petiolaris } \\
\text { Mart. }\end{array}$ & 0,01 & 0,015 & 0,108 & 0,023 & 0,04 & - & - & - & 0,196 \\
\hline $\begin{array}{l}\text { Byrsonima coccolobifolia } \\
\text { Kunth. }\end{array}$ & 0,055 & 0,058 & 0,019 & 0,063 & - & - & - & - & 0,195 \\
\hline $\begin{array}{l}\text { Strychnos pseudoquina } \\
\text { A.St.-Hil. }\end{array}$ & - & 0,008 & 0,018 & - & 0,163 & - & - & - & 0,188 \\
\hline $\begin{array}{l}\text { Enterolobium } \\
\text { gummiferum (Mart.) J. F. } \\
\text { Macbr. }\end{array}$ & 0,001 & - & 0,04 & 0,062 & - & - & 0,061 & - & 0,164 \\
\hline Eugenia dysenterica D. C. & 0,031 & 0,063 & 0,049 & - & - & - & - & - & 0,142 \\
\hline $\begin{array}{l}\text { Erythroxylum deciduum } \\
\text { A.St.-Hil. }\end{array}$ & 0,049 & 0,028 & 0,039 & - & - & - & - & - & 0,115 \\
\hline $\begin{array}{l}\text { Eryotheca gracilipes (K. } \\
\text { Schum.) }\end{array}$ & 0,008 & 0,008 & - & 0,032 & 0,063 & - & - & - & 0,111 \\
\hline $\begin{array}{l}\text { Handroanthus ochraceus } \\
\text { (Cham.) Standl. }\end{array}$ & 0,033 & 0,038 & 0,03 & - & - & - & - & - & 0,101 \\
\hline $\begin{array}{l}\text { Agonandra brasiliensis } \\
\text { Miers ex Benth. \& Hook. } \\
\text { f. }\end{array}$ & 0,038 & 0,049 & 0,012 & - & - & - & - & - & 0,098 \\
\hline Mouriri elliptica Mart. & 0,009 & 0,051 & 0,021 & - & - & - & - & - & 0,081 \\
\hline Rourea induta Planch & 0,031 & 0,021 & 0,012 & - & - & - & - & - & 0,064 \\
\hline $\begin{array}{l}\text { Erythroxylum suberosum } \\
\text { A.St.-Hil. }\end{array}$ & 0,036 & 0,013 & - & - & - & - & - & - & 0,05 \\
\hline Heisteria ovata Benth. & 0,005 & 0,018 & - & 0,022 & - & - & - & - & 0,045 \\
\hline $\begin{array}{l}\text { Psidium myrsinites Mart. } \\
\text { Ex DC. }\end{array}$ & 0,012 & 0,011 & 0,022 & - & - & - & - & - & 0,045 \\
\hline $\begin{array}{l}\text { Byrsonima verbascifolia } \\
\text { (L) Rich. }\end{array}$ & 0,007 & 0,034 & - & - & - & - & - & - & 0,04 \\
\hline
\end{tabular}

(L) Rich. 
Tabela 7 - Conclusão...

Table 7 -Conclusion...

\begin{tabular}{|c|c|c|c|c|c|c|c|c|c|}
\hline \multirow[t]{2}{*}{ Espécies } & \multicolumn{9}{|c|}{ Classes diamétricas $\mathrm{d} 30 \mathrm{~cm}(\mathrm{~cm})$} \\
\hline & 58,9 & $9-12,9$ & $13-16,9$ & 17-20,9 & $21-24,9$ & $25-28,9$ & 29-32,9 & $>33$ & $\sum$ \\
\hline $\begin{array}{l}\text { Tabebuia aurea (Silva } \\
\text { Manso) Benth. \& Hook.f. } \\
\text { ex S. Moore }\end{array}$ & 0,002 & 0,03 & - & - & - & - & - & - & 0,032 \\
\hline $\begin{array}{l}\text { Acosmium dasycarpum } \\
\text { (Vog.) Yakovl. }\end{array}$ & 0,006 & 0,023 & - & - & - & - & - & - & 0,029 \\
\hline Byrsonima sp.1 & 0,012 & - & 0,016 & - & - & - & - & - & 0,028 \\
\hline $\begin{array}{l}\text { Buchenavia tomentosa } \\
\text { Eichl. }\end{array}$ & - & 0,005 & - & 0,019 & - & - & - & - & 0,025 \\
\hline $\begin{array}{l}\text { Vatairea macrocarpa } \\
\text { (Benth.) Ducke }\end{array}$ & 0,003 & - & 0,017 & - & - & - & - & - & 0,02 \\
\hline Qualea grandiflora Mart. & 0,004 & 0,01 & 0,005 & - & - & - & - & - & 0,019 \\
\hline Annona crassiflora Mart. & - & 0,005 & 0,01 & - & - & - & - & - & 0,015 \\
\hline $\begin{array}{l}\text { Myrcia guianensis (Aubl.) } \\
\text { DC }\end{array}$ & 0,01 & 0,004 & - & - & - & - & - & - & 0,014 \\
\hline Byrsonima sp. 2 & 0,005 & 0,006 & - & - & - & - & - & - & 0,011 \\
\hline $\begin{array}{l}\text { Stryphnodendron } \\
\text { adstrigens (Mart.) }\end{array}$ & 0,002 & 0,006 & - & - & - & - & - & - & 0,009 \\
\hline Vochysea rufa Mart. & - & 0,008 & - & - & - & - & - & - & 0,008 \\
\hline $\begin{array}{l}\text { Psidium pohlianum O. } \\
\text { Berg }\end{array}$ & 0,002 & 0,004 & - & - & - & - & - & - & 0,006 \\
\hline $\begin{array}{l}\text { Eugenia ovalifolia } \\
\text { Cambess }\end{array}$ & - & 0,006 & - & - & - & - & - & - & 0,006 \\
\hline $\begin{array}{l}\text { Dimorphandra mollis } \\
\text { Benth. }\end{array}$ & - & 0,005 & - & - & - & - & - & - & 0,005 \\
\hline Palicourea rigida Kunth & 0,005 & - & - & - & - & - & - & - & 0,005 \\
\hline Neea theifera Oerst. & 0,005 & - & - & - & - & - & - & - & 0,005 \\
\hline $\begin{array}{l}\text { Aspidosperma } \\
\text { macrocarpa Mart. }\end{array}$ & - & 0,005 & - & - & - & - & - & - & 0,005 \\
\hline Acosmium sp. & 0.004 & - & - & - & - & - & - & - & 0,004 \\
\hline $\begin{array}{l}\text { Hancornia speciosa } \\
\text { Gomes }\end{array}$ & - & 0,004 & - & - & - & - & - & - & 0,004 \\
\hline Eugenia sp1. & - & 0,003 & - & - & - & - & - & - & 0,003 \\
\hline Tachigali paniculata Aubl. & 0,002 & - & - & - & - & - & - & - & 0,002 \\
\hline $\begin{array}{l}\text { Tocoyena formosa } \\
\text { (Cham. \& Schltdl.)K. }\end{array}$ & 0,002 & - & - & - & - & - & - & - & 0,002 \\
\hline Rourea sp. & 0,002 & - & - & - & - & - & - & - & 0,002 \\
\hline $\begin{array}{l}\text { Aegiphila ihotzkiana } \\
\text { Cham. }\end{array}$ & 0,002 & - & - & - & - & - & - & - & 0,002 \\
\hline Eugenia sp. 2 & 0,001 & - & - & - & - & - & - & - & 0,001 \\
\hline Miconia pohliana Cogn. & 0,001 & - & - & - & - & - & - & - & 0,001 \\
\hline Erythroxylum sp. & 0 & - & - & - & - & - & - & - & 0 \\
\hline$\sum$ (Mg ha-1) & 1,601 & 2,818 & 2,251 & 1,052 & 1,037 & 0,594 & 0,435 & 1,12 & 10,908 \\
\hline$\%$ do total & 14,68 & 25,83 & 20,63 & 9,64 & 9,51 & 5,44 & 3,99 & 10,27 & 100 \\
\hline
\end{tabular}

* Espécies de maior valor de importância no cerrado típico estudado (OLIVEIRA et al., 2015). 
Estoques de biomassa seca e carbono do componente herbáceo-arbustivo

Na vegetação herbácea-arbustiva, o estoque médio de biomassa seca foi estimado em 3,06 $\mathrm{Mg} \mathrm{ha}^{-1}$ correspondendo a um estoque médio de $1,44 \mathrm{Mg}^{-1}$ de carbono neste compartimento (Tabela 8). Poucos trabalhos de quantificação de estoques de biomassa e carbono em cerrado sensu stricto apresentaram estimativas para o compartimento herbáceo-arbustivo. Ribeiro et al. (2011), encontraram para um cerrado sensu stricto em Minas Gerais estoque médio de 4,68 $\mathrm{Mg} \mathrm{ha}^{-1}$ de biomassa seca na vegetação lenhosa arbustiva. Em estudo realizado por Abdala et al. (1998), verificou-se que o estrato herbáceo-arbustivo de um cerrado sensu stricto no Distrito Federal estoca em média 5,58 $\mathrm{Mg} \mathrm{ha}^{-1}$ de biomassa seca, das quais 4,13 $\mathrm{Mg}^{-1}$ ha correspondem à biomassa de gramíneas, que responde por $74 \%$ do estoque neste compartimento. Conforme estes autores, nos cerrados, a proporção de gramíneas varia de 50 a $80 \%$, dependendo da densidade da cobertura arbórea, estação de amostragem e regime do fogo. No compartimento herbáceo-arbustivo do cerrado típico estudado, os componentes gravetos e folhas responderam por $63 \%$ do estoque total do compartimento, enquanto as gramíneas foram responsáveis por $37 \%$ (Tabela 8 ).

Tabela 8 - Estoques de biomassa seca nos diferentes componentes do compartimento herbáceoarbustivo.

Table 8 - Stocks in dry biomass and carbon in the different components of the herbaceous-shrubby compartment.

\begin{tabular}{lcccccc}
\hline Variável & Média & $\mathbf{S}_{\mathbf{y}} \mathbf{t}$ & $\left.\mathbf{( S}_{\mathbf{y}} \mathbf{t}\right) \%$ & Limite Inferior & Limite Superior & \% do Total \\
\hline Biomassa -Total $\left(\mathrm{Mg} \mathrm{ha}^{-1}\right)$ & 3,06 & 0,64 & 20,77 & 2,43 & 3,7 & 100 \\
Biomassa-Gramíneas $\left(\mathrm{Mg} \mathrm{ha}^{-1}\right)$ & 1,14 & 0,25 & 21,56 & 0,89 & 1,38 & 37 \\
Biomassa - Gravetos $\left(\mathrm{Mg} \mathrm{ha}^{-1}\right)$ & 1,16 & 0,45 & 39,15 & 0,7 & 1,61 & 38 \\
Biomassa- Folhas $\left(\mathrm{Mg} \mathrm{ha}^{-1}\right)$ & 0,77 & 0,27 & 35,19 & 0,5 & 1,04 & 25 \\
\hline
\end{tabular}

Em que: $\mathrm{S}_{\bar{y}} \mathrm{t}=$ Erro amostral em termos absolutos; $\left(\mathrm{S}_{\bar{y}} \mathrm{t}\right) \%=$ Erro amostral em termos relativos. $(\alpha=0,05)$

Estoques de biomassa seca e carbono da serapilheira

A biomassa seca estocada na serapilheira foi estimada em 4,81 $\mathrm{Mg} \mathrm{ha}^{-1}$ correspondendo a um estoque médio de 2,13 $\mathrm{Mg} \mathrm{ha}^{-1}$ de carbono neste compartimento (Tabela 9).

Tabela 9 - Estoques de biomassa seca e carbono na serapilheira.

Table 9 - Stocks in dry biomass and carbon in the litter.

\begin{tabular}{lccccc}
\hline Variável & Média & $\mathbf{S}_{\bar{y}} \mathbf{t}$ & $\left(\mathbf{S}_{\bar{y}} \mathbf{t}\right) \mathbf{\%}$ & Limite Inferior & Limite Superior \\
\hline Biomassa seca $\left(\mathrm{Mg} \mathrm{ha}^{-1}\right)$ & 4,81 & 1,55 & 15,4 & 3,26 & 6,36 \\
Carbono $\left(\mathrm{Mg} \mathrm{ha}^{-1}\right)$ & 2,13 & 0,69 & 15,4 & 1,45 & 2,82 \\
\hline
\end{tabular}

Em que: $\mathrm{S}_{\overline{\mathrm{y}}} \mathrm{t}=$ Erro amostral em termos absolutos; $\left(\mathrm{S}_{\overline{\mathrm{y}}} \mathrm{t}\right) \%=$ Erro amostral em termos relativos. $(\alpha=0,05)$

Paiva, Rezende e Pereira (2011) encontraram estoque médio de 3,62 $\mathrm{Mg}^{\mathrm{a}}{ }^{-1}$ de carbono na serapilheira de um cerrado sensu stricto. Morais (2012) encontrou estoque médio de 3,05 $\mathrm{Mg} \mathrm{ha}^{-1} \mathrm{de}$ carbono armazenadas na serapilheira de cerrado sensu stricto na sub-bacia do Rio São Francisco no norte de Minas Gerais. Dois fragmentos estudados por Morais (2012) apresentaram estoques de carbono próximos aos encontrados no presente estudo, sendo observados valores médios de $1,04 \mathrm{Mg} \mathrm{ha}^{-1}$ e 2,04 $\mathrm{Mg} \mathrm{ha}^{-1}$. Ribeiro et al. (2011) verificaram média de 6,31 $\mathrm{Mg} \mathrm{ha}^{-1}$ para o estoque de biomassa seca neste 
compartimento em um cerrado sensu stricto de Minas Gerais. Observa-se, portanto, que os estoques de carbono na serapilheira de cerrados sensu stricto variam na ordem de 1,04 $\mathrm{Mg} \mathrm{ha}^{-1}$ a 3,62 $\mathrm{Mg} \mathrm{ha}^{-1}$, em conformidade com trabalhos realizados nesta fisionomia, e que os valores observados no presente estudo se encontram dentro destes limites de variação, tendendo ao limite inferior.

\section{Estoques totais de biomassa e carbono}

A biomassa acima do solo total na área de estudada foi estimada em $18,77 \mathrm{Mg} \mathrm{ha}^{-1}$, correspondendo ao estoque total de $8,67 \mathrm{Mg} \mathrm{ha}^{-1} \mathrm{de}$ carbono, em que o compartimento arbóreo foi responsável pela maior parte dos estoques (58\%), seguido da serapilheira (26\%) e herbáceo-arbustivo (16\%).

A biomassa acima do solo total, em cerrado sensu stricto, varia de $12,55 \mathrm{Mg} \mathrm{ha}^{-1}$ a $76,63 \mathrm{Mg} \mathrm{ha}^{-1}$, quando se consideram os compartimentos arbóreo, herbáceo-arbustivo e serapilheira (ABDALA et al., 1998; CASTRO; KAUFFMAM, 1998; OTTMAR, 2001; RIBEIRO et al., 2011). Contudo, poucos são os trabalhos que buscaram até o momento quantificar os estoques nesses diferentes compartimentos, sendo as metodologias de estimativas muito variadas nos trabalhos existentes. Para o cerrado sensu stricto no Distrito Federal, Abdala et al. (1998) verificaram estoque acima do solo total de $39,8 \mathrm{Mg} \mathrm{ha}^{-1}$ de biomassa. Considerando compartimento arbóreo, serapilheira e arbustos lenhosos, Ribeiro et al. (2011) observaram para o cerrado sensu stricto em Minas Gerais estoque total de biomassa acima do solo de 76,63 $\mathrm{Mg} \mathrm{ha}^{-1}$. Estimativas realizadas por Ottmar et al. (2001) mostraram para cinco áreas de cerrado sensu stricto, biomassa acima do solo total variando de $20,9 \mathrm{Mg}$ $\mathrm{ha}^{-1}$ a 58,01 Mg ha-1. Conforme Castro e Kauffmam (1998), a biomassa acima do solo total (incluindo árvores, estrato herbáceo-arbustivo, e serapilheira) tende a ser maior à medida que a vegetação tende a ser mais densa. Os autores verificaram que a biomassa aumentou ao longo do gradiente de 5,5 $\mathrm{Mg} \mathrm{ha}^{-1}$ para campo limpo a $29,4 \mathrm{Mg} \mathrm{ha}^{-1}$ no cerrado denso. Deste modo, os valores observados encontram-se no intervalo verificado para outras áreas de cerrado estudadas no Brasil, e apontam que os estoques de biomassa e carbono para cerrados influenciados por arenitos parece estar no limite inferior deste intervalo.

\section{Conclusão}

Com base nas medidas de precisão e distribuição residual, a equação proveniente do modelo de Schumacher \& Hall (logarítmica) foi escolhida para estimar os estoques de biomassa seca do compartimento arbóreo do cerrado típico. A biomassa aérea total foi de 18,77 $\mathrm{Mg} \mathrm{ha}^{-1}$, correspondendo a um estoque total de $8,67 \mathrm{Mg} \mathrm{ha}^{-1}$ de carbono, no qual o compartimento arbóreo (56\%), foi responsável pela maior parte dos estoques, seguido da serapilheira (25\%) e herbáceo-arbustivo (19\%). Os valores observados encontram-se no intervalo verificado para outras áreas de Cerrado estudadas no Brasil, apontando que Cerrados influenciados pela Formação Urucuia parecem estar no limite inferior deste intervalo, assemelhando-se com áreas amostradas sobre Neossolos Quartzarênicos.

\section{Referências}

ABDALA, G. C. et al. Above and belowground organic matter and root: shoot ratio in a Cerrado in central Brazil. Brazilian Journal of Ecology, Rio Claro, v. 2, n. 1, p. 11-23, 1998.

BRASIL. Ministério do Meio Ambiente. Plano de ação para prevenção e controle do desmatamento e das queimadas: cerrado. Brasília: MMA, 2011. 200 p.

CASTRO, E. A.; KAUFFMAN, J. B. Ecosystem structure in the Brazilian Cerrado: a vegetation gradient of aboveground biomass, root mass and consumption by fire. Journal of Tropical Ecology, Cambridge, v. 14, p. 263-283, may 1998.

CASTRO, K. B. et al. Caracterização Geomorfológica do Município de Jaborandi, Oeste baiano, escala 1:100.000. Planaltina: EMBRAPA Cerrados, 2010. 32 p. 
DIAS, B. F. S. Conservação da biodiversidade no bioma Cerrado: histórico dos impactos antrópicos no bioma Cerrado. In: FALEIRO, F. G.; FARIAS NETO, A. L. (Org.). Savanas: desafios e estratégias para o equilíbrio entre sociedade, agronegócio e recursos naturais. Planaltina: EMBRAPA Cerrados, 2008. p. 303-33.

FELFILI, J. M. et al. Diversity, floristics and structural patterns of cerrado vegetation in central Brazil. Plant Ecology, Dordrecht, v. 175, p. 37-46, jul. 2004.

FELFILI, M. C. Proposição de critérios florísticos, estruturais e de produção para o manejo de cerrado sensu stricto do Brasil central. 2008. 133 f. Tese (Doutorado em Ciências Florestais) - Universidade de Brasília, Brasília, 2008.

HARIDASAN, M. Competição por nutrientes em espécies arbóreas do cerrado. In: SCARIOT, A.; SOUSA-SILVA, J. C.; FELFILI, J. M. (Org.). Cerrado: ecologia, biodiversidade e conservação. Brasília: Ministério do Meio Ambiente, 2005. p. 167-178.

INTERGOVERNMENTAL PANEL ON CLIMATE CHANGE. Guidelines for national greenhouse gas inventories, prepared by the National Greenhouse Gas Inventories Programme. Japan: IGES, 2006.

KLINK, C. A.; MACHADO, R. B. A conservação do Cerrado brasileiro. Megadiversidade, Belo Horizonte, v. 1, n. 1, p. 147-155, jul. 2005.

MORAIS, V. A. Modelagem e espacialização do estoque de carbono de cerrado sensu stricto em Minas Gerais. 2012. 124 f. Dissertação (Mestrado em Ciências Florestais) - Universidade Federal de Lavras, Lavras. 2012.

OLIVEIRA, C. P. et al. Composição florística e estrutura de um Cerrado sensu stricto no oeste da Bahia. CERNE, Lavras, v. 21, n. 4, p. 545-552, set. 2015.

OTTMAR, R. D. et al. Stereo photo series for quantifying cerrado fuels in Central Brazil- Volume I. Portland: Department of Agriculture, Forest Service, Pacific Northwest Research Station, 2001. 87 p.

PAIVA, A. O.; REZENDE, A. V.; PEREIRA, R. S. Estoque de Carbono em Cerrado Sensu stricto no Distrito Federal. Revista Árvore, Viçosa, MG, v. 35, n. 3, p. 527-538, abr. 2011.

REZENDE, A. V. et al. Comparação de modelos matemáticos para estimativa do volume, biomassa e estoque de carbono da vegetação lenhosa de um cerrado sensu stricto em Brasília, DF. Scientia Florestales, Piracicaba, n. 71, p. 65-76, ago. 2006.

RIBEIRO, S. C. et al. Above- and belowground biomass in a Brazilian Cerrado. Forest Ecology and Management, Amsterdam, v. 262, n. 3, p. 491-499, apr. 2011.

SCOLFORO, J. R. S. Biometria florestal: Parte I: Modelos de regressão linear e não linear; Parte II: Modelos para relação hipsométrica, volume, afilamento e peso de matéria seca. Lavras: UFLA; FAEPE, 2005. $352 \mathrm{p}$.

SCOLFORO, J. R. S.; OLIVEIRA, A. D.; ACERBI JÚNIOR, F. W. Inventário Florestal de Minas Gerais: equações de volume, peso de matéria seca e carbono para diferentes fitofisionomias da flora nativa. Lavras: Editora UFLA, 2008. 216 p.

SILVA, R. B. M. et al. Relação solo/vegetação em ambiente de Cerrado sobre influência do Grupo Urucuia. Ciência Florestal, Santa Maria, v. 25, n. 2, p. 363-373, abr./jun. 2015. 